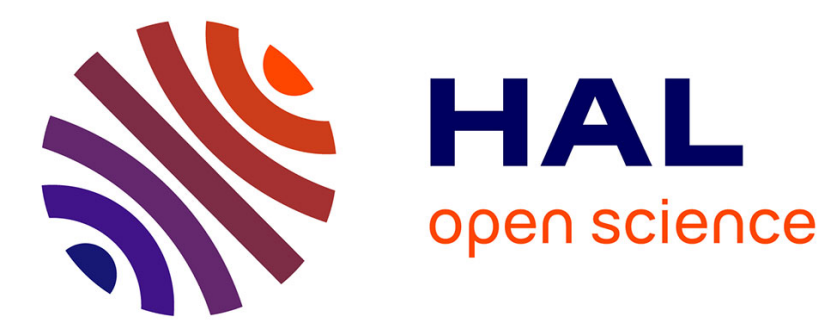

\title{
Generalized theory of acoustic propagation in porous dissipative media
}

Maurice A. Biot

\section{To cite this version:}

Maurice A. Biot. Generalized theory of acoustic propagation in porous dissipative media. Journal of the Acoustical Society of America, 1962, 34 (9), pp.1254-1264. 10.1121/1.1918315 . hal-01368727

\section{HAL Id: hal-01368727 \\ https://hal.science/hal-01368727}

Submitted on 21 Sep 2016

HAL is a multi-disciplinary open access archive for the deposit and dissemination of scientific research documents, whether they are published or not. The documents may come from teaching and research institutions in France or abroad, or from public or private research centers.
L'archive ouverte pluridisciplinaire HAL, est destinée au dépôt et à la diffusion de documents scientifiques de niveau recherche, publiés ou non, émanant des établissements d'enseignement et de recherche français ou étrangers, des laboratoires publics ou privés. 


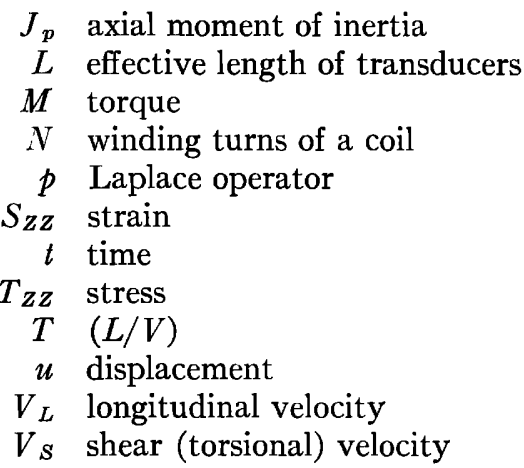

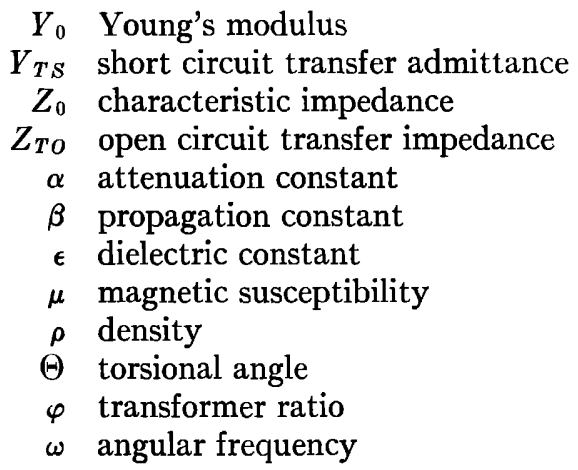

\title{
Generalized Theory of Acoustic Propagation in Porous Dissipative Media*
}

\author{
M. A. Bior \\ Shell Development Company, Houston 1, Texas
}

(Received November 3, 1961)

\begin{abstract}
The theory of acoustic propagation in porous media is extended to include anisotropy, viscoelasticity, and solid dissipation. A more refined analysis of the relative motion of the fluid in the pores is also developed by introducing the concept of viscodynamic operational tensor. The nature of this operator is analyzed by applying variational and Lagrangian methods. Viscoelasticity and solid dissipation are introduced by applying the correspondence principle as derived from thermodynamics in earlier work by the author. Various dissipative models are discussed and the corresponding operators and relaxation spectra are derived. The physical chemistry of the multiphase porous medium including surface effects lies within the scope of the thermodynamic theory. The nature of thermoelastic dissipation and electrokinetic effects in relation to the thermodynamic theory is also brought out.
\end{abstract}

\section{INTRODUCTION}

$T$ HE propagation of acoustic waves in porous media has been the object of theoretical analyses by various authors. ${ }^{1-4} \mathrm{~A}$ more systematic treatment of acoustic propagation in a porous elastic matrix containing a viscous fluid has been given by the writer. ${ }^{5,6}$ It

* A short outline of the present paper was presented under the title "Théorie generalisée de la propagation acoustique dans un solide poreux dissipatif" at the Colloquium on Propagation in Nonhomogeneous Media, CNRS, Marseilles, September 11-16, 1961, and published in the CNRS Proceedings No. 111, pp. 5765 (1962).

${ }^{1} \mathrm{~J}$. Frenkel, "On the Theory of Seismic and Seismoelectric Phenomena in Moist Soils," J. Phys. U.S.S.R. 8, 230 (1944).

${ }^{2}$ C. Zwikker and C. W. Kosten, Sound Absorbing Materials (Elsevier Publishing Company, Inc., New York, 1949).

${ }^{3}$ L. L. Beranek, "Acoustical Properties of Homogeneous, Isotropic Rigid Tiles and Flexible Blankets," J. Acoust. Soc. Am. 19, 556-568 (1947).

' R. W. Morse, "Acoustic Propagation in Granular Media," J. Acoust. Soc. Am. 24, 696-700 (1952).

5 M. A. Biot, "Theory of Propagation of Elastic Waves in a Fluid-Saturated Porous Solid-I. Low Frequency Range," J. Acoust. Soc. Am. 28, 168-178 (1956).

${ }^{6}$ M. A. Biot, "Theory of Propagation of Elastic Waves in a Fluid-Saturated Porous Solid-II. Higher Frequency Range," J. Acoust. Soc. Am. 28, 179-191 (1956). was derived by adding inertia terms into the equations of consolidation developed in earlier theories. ${ }^{7,8}$

An extension of the acoustic propagation theory to include anisotropy and viscoelasticity was briefly outlined recently in the wider context of the mechanics of porous media. ${ }^{9}$ In the present treatment the new features of the theory are developed in more detail and further generalized by the introduction of a "viscodynamic operator." This provides an exact procedure for the evaluation of the dynamic properties of the fluid in its motion relative to the solid. While it constitutes an important refinement, it also leads to a new and simplified derivation of the fundamental equations of propagation. In addition, a more detailed analysis is given of viscoelastic and solid dissipation, and a number of models are discussed as illustrative examples.

7 M. A. Biot, "General Theory of Three-Dimensional Consolidation," J. Appl. Phys. 12, 155-164 (1941).

${ }^{8}$ M. A. Biot, "General Solutions of the Equations of Elasticity and Consolidation for a Porous Material," J. Appl. Mech., Trans. Am. Soc. Mech. Engrs. 78, 91-96 (1956).

${ }_{9}$ M. A. Biot, "The Mechanics of Deformation and Acoustic Propagation in Porous Media," J. Appl. Phys. 33, 1482-1498 (1962). 
Attention is called to the inclusion of physical-chemical phenomena into the present theory.

The concept of a viscodynamic operator, which is introduced in Sec. $\amalg$, offers the advantage of embodying the dynamic features of the fluid motion in the pores. A major portion of the theory is thereby reduced to an evaluation of the viscodynamic operator. Various methods for the evaluation of this operator are discussed in Secs. III, IV, and V. A scaled-model test is suggested. This model is applicable for fluid velocities which are beyond the range of validity of the present theory, causing amplitude-dependent effects which are of hydrodynamic origin and involve the Reynolds number. Analytic procedures are also developed. In particular it is shown that Lagrangian and variational techniques including the the use of normal coordinates may be applied to derive exact or approximate expressions for the viscodynamic operator. Such methods yield results which are valid in both the low- and high-frequency ranges. It is also shown that in anisotropic media the viscodynamic operator is a second-rank symmetric tensor and that its principal directions may involve different characteristic frequencies.

An interesting result is obtained by the use of Lagrangian equations. In Sec. III it is shown that use of the Poiseuille microvelocity field for the evaluation of the kinetic energy and the dissipation function yields results which are satisfactory in the low-frequency range up to frequencies where the viscous and inertia forces are of the same order. For a pore size of $10^{-2} \mathrm{~cm}$ and water at normal temperature, this frequency is about 300 cps. The viscoelastic properties, relaxation effects, and solid dissipation are included in the theory by applying the equation of deformations of a porous medium derived earlier from the thermodynamics of irreversible processes..$^{10}$ The formulation is based on the correspondence principle proposed by the writer in its general form and shown to be valid for porous media. ${ }^{10}$ We have discussed various dissipative models which involve intergranular effects, small fluid-filled cracks, relaxation effects due to fluid bulk viscosity or caused by nonequilibrium processes of chemical and physical nature at fluid-solid interfaces. The dissipative effect of microscopic air bubbles is also discussed. This brings in the additional feature of inertia forces in the "internal" coordinates. All these models are translated into specific operators as particular cases of the general thermodynamic theory.

More specifically in Sec. VI attention is called to the behavior of contact areas between grains and the behavior of fluids in the microscopic spaces in regions of quasi-contact and cracks. In such regions the physical chemistry of surface effects and special intermolecular forces will generally play an important role and contribute to the over-all rigidity and dissipation effects. These phenomena are included in the present

\footnotetext{
${ }^{10}$ M. A. Biot, "Theory of Deformation of a Porous Viscoelastic Anisotropic Solid,” J. Appl. Phys. 27, 459-467 (1956).
}

treatment and may be evaluated thermodynamically if we know the free energy and dissipation function of the physical-chemical system.

The acoustic propagation is assumed to be associated with amplitudes small enough so that intergranular dissipation remains in the linear range. In that case the dissipation is of the relaxation type and should fall within the scope of linear thermodynamics. However extension into the nonlinear range is possible as an empirical procedure by introducing an amplitude dependence into the parameters of the linear theory.

It is to be expected that the phenomena associated with the properties of microscopic fluid gaps play an important part in the variation of acoustic properties of porous rock when tested under high and low effective pressure.

Thermoelastic dissipation is briefly mentioned and it is indicated that it is included in the present analysis and may be expressed by the use of the operators of the same thermodynamic theory.

Electrokinetic effects and the associated acoustic dissipation are also discussed. Two types of effects are considered. One of these may be included directly in the present treatment. The other type may be evaluated by the use of existing theories. ${ }^{1}$ It is pointed out that a more systematic treatment leading to coupled equations for the electrical and mechanical variables may be derived from the thermodynamic principles.

Dissipation due to scatter is neglected; therefore, it must be assumed that the wavelength is large relative to the pore size.

\section{FUNDAMENTAL EQUATIONS}

Consider a porous elastic matrix, fluid-saturated and statistically isotropic. The stress-strain relations derived in previous work ${ }^{1,2}$ are

$$
\begin{aligned}
\tau_{i j} & =2 \mu e_{i j}+\delta_{i j}\left(\lambda_{c} e-\alpha M \zeta\right), \\
p_{f} & =-\alpha M e+M \zeta .
\end{aligned}
$$

The physical quantities in these equations are defined as follows:

$$
\begin{aligned}
\mathbf{u}= & \text { displacement of solid of components } u_{i}, \\
\mathbf{w}= & \text { displacement of fluid relative to solid of com- } \\
& \text { ponents } w_{i} \text { (measured in volume per unit area), } \\
e_{i j}= & \frac{1}{2}\left(\partial u_{i} / \partial x_{j}+\partial u_{j} / \partial x_{i}\right) \\
e= & \text { divu, } \\
\zeta= & - \text { divw, } \\
p_{f}= & \text { fluid pressure in the pores, } \\
\tau_{i j}= & \text { total stress in the medium, } \\
\delta_{i j}= & \text { Kronecker symbol. }
\end{aligned}
$$

The other quantities in Eqs. (2.1) are elastic coefficients. A detailed derivation and discussion of Eqs. (2.1) has also been given in a recent paper. $^{9}$ 
Dynamical equations were derived in previous papers by various methods..$^{3-5} \mathrm{~A}$ new and more general form of these equations is obtained as follows. One of the dynamical equations expresses the time derivative of total momentum of the mixed fluid-solid system and is written

$$
\partial \tau_{i j} / \partial x_{j}=\rho \ddot{u}_{i}+\rho_{f} \ddot{w}_{i},
$$

where $\rho$ is the mass density of the mixed medium and $\rho_{f}$ that of the fluid.

A second equation expresses the law of relative motion of the fluid in the pores, i.e.,

$$
-\partial p_{f} / \partial x_{i}-\rho_{f} \ddot{u}_{i}=\bar{Y}(p) \dot{w}_{i} .
$$

The left side represents the effective body force per unit volume acting on the fluid in relative motion. The operator $\bar{Y}(p)$ is a function of the differential operator

$$
p=d / d t \text {. }
$$

For simple harmonic motion at the angular frequency $\omega$, this quantity becomes a pure imaginary

$$
p=i \omega \text {. }
$$

The operator $\bar{Y}$ embodies the dynamics of the fluid in relative motion. We shall refer to it as the viscodynamic operator. It depends on the fluid inertia, its viscosity, and the geometry of the pores. Its evaluation is discussed below in Secs. III and IV for the isotropic medium and in Sec. V for the anisotropic case.

If we substitute the values (2.1) for the stress into Eqs. (2.2) and (2.3), we obtain

$$
\begin{aligned}
\mu \nabla^{2} \mathbf{u}+\operatorname{grad}\left[\left(\mu+\lambda_{c}\right) e-\alpha M \zeta\right] & =\left(\partial^{2} / \partial t^{2}\right)\left(\rho \mathbf{u}+\rho_{f} \mathbf{w}\right) \\
\operatorname{grad}(\alpha M e-M \zeta) & =\left(\partial^{2} / \partial t^{2}\right)\left(\rho_{f} \mathbf{u}\right) \\
& +\bar{Y} \partial \mathbf{w} / \partial t
\end{aligned}
$$

These equations for acoustic propagation are written here in a form corresponding to the case of uniform rigidity ( $\mu=$ const).

\section{VISCODYNAMIC OPERATOR IN THE LOW-FREQUENCY RANGE}

In previous work ${ }^{3-5}$ we have formulated the dynamical equations directly in Lagrangian form. The same Lagrangian procedure is used here to derive the operator $\bar{Y}$. This is done by introducing the dissipation function

$$
D=(\eta / 2 k)\left(\dot{w}_{x}^{2}+\dot{w}_{y}^{2}+\dot{w}_{z}^{2}\right)
$$

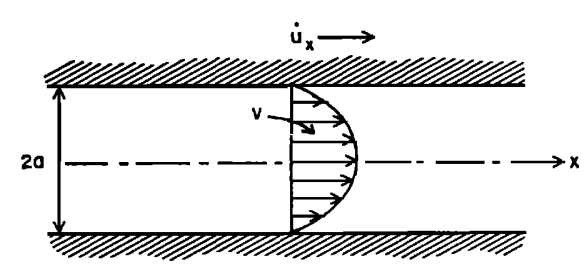

FIG. 1. Two-dimensional Poiseuille flow between parallel boundaries. and the kinetic energy of the fluid in relative motion

$$
\tau=\frac{1}{2} m\left(\dot{w}_{x}^{2}+\dot{w}_{y}{ }^{2}+\dot{w}_{z}{ }^{2}\right) \text {. }
$$

The fluid viscosity is denoted by $\eta$ and $k$ is Darcy's coefficient of permeability. The coefficient $m$ is derived by integrating the kinetic energy of the microvelocity field of the fluid in relative motion. In carrying out these evaluations we assume that the microvelocity field is the same as in Poiseuille flow. This is equivalent to stating that they correspond to the case where the inertia forces are neglected. The components $v_{i}$ of this velocity field may be written as linear functions of the vector $\mathbf{w}$

$$
v_{i}=\alpha_{i j} \dot{w}_{j},
$$

where $\alpha_{i j}$ depends on the coordinates. The dissipation function is

$$
D=\frac{1}{2} \eta \iiint_{\Omega} \epsilon_{i j} \epsilon_{i j} d \Omega,
$$

where the volume integral extends to the fluid in the unit volume of mixed material and the microstrain rate in the fluid is

or

$$
\epsilon_{i j}=\frac{1}{2}\left(\partial v_{i} / \partial x_{j}+\partial v_{j} / \partial x_{i}\right),
$$

$$
\epsilon_{i j}=\frac{1}{2}\left(\partial \alpha_{i k} / \partial x_{j}+\partial \alpha_{j k} / \partial x_{i}\right) \dot{w}_{k} .
$$

Substituting this value in the integral and assuming that the medium is statistically isotropic, we find an expression of the type (3.1). As already pointed out elsewhere, ${ }^{9,10}$ this calculation amounts to a derivation of Darcy's coefficients from thermodynamic principles. In this connection it should be noted as already pointed out earlier ${ }^{9}$ that the thermodynamic derivation is applicable to the much more general case where the entropy production involves other mechanisms than purely viscous friction such as slip flow or more complex interfacial effects.

Similarly the kinetic energy is written

$$
\tau=\frac{1}{2} \rho_{f} \dot{w}_{i} \dot{w}_{j} \iiint_{\Omega} \alpha_{k i} \alpha_{k j} d \Omega
$$

which yields the coefficient $m$.

The Lagrangian equations in relative motion are written

or

$$
-\frac{\partial p_{f}}{\partial x_{i}}-\rho_{f} \ddot{u}_{i}=\frac{\partial D}{\partial \dot{w}_{i}}+\frac{d}{d t}\left(\frac{\partial T}{\partial \dot{w}_{i}}\right),
$$

$$
-\partial p_{f} / \partial x_{i}-\rho_{f} \ddot{u}_{i}=(\eta / k) \dot{w}_{i}+m \ddot{w}_{i} .
$$

Comparing with Eq. (2.3) this yields, for the viscodynamic operator,

$$
\bar{Y}(p)=(\eta / k)+m p .
$$

At zero frequency $(p=0)$ the operator yields Darcy's law. 
The microvelocity field used in the above derivation is that of Poiseuille flow which implies that the inertia forces are negligible in comparison with the effect of the viscosity. One may well ask how valid Eq. (3.10) remains at increasing frequencies. One answer to this question is readily obtained by comparison with an exact solution already evaluated previously. Consider a fluid confined between two parallel plane boundaries separated by a distance $2 a$ (Fig. 1). The problem of the relative motion of this fluid parallel to the boundaries under oscillatory driving forces is well known. In a previous paper ${ }^{6}$ it was derived in the form

$$
-\frac{\partial p_{f}}{\partial x}-\rho_{f} \ddot{u}_{x}=\frac{\eta}{a^{2}} \frac{z^{2}}{1-(1 / z) \tanh z} \dot{w},
$$

where

$$
z=a(\rho / \nu)^{\frac{1}{2}}, \quad \nu=\eta / \rho_{f} .
$$

The flow field is two-dimensional and in the plane of the figure. The average velocity of the fluid relative to the wall is

$$
v_{\mathrm{av}}=\dot{w}
$$

The wall displacement is denoted by $u_{x}$ and $d p_{f} / d x$ is the pressure gradient along the axis. The viscodynamic operator for this case is

$$
\bar{Y}(p)=\frac{\eta}{a^{2}} \frac{z^{2}}{1-(1 / z) \tanh z} .
$$

We may put this in the form

$$
\bar{Y}(p)=\left(3 \eta / a^{2}\right)\left(F_{1}+\frac{1}{3} z^{2}\right),
$$

where $F_{1}$ has been evaluated as a function of $z$ in an earlier paper. ${ }^{11}$

Now this same problem may be solved by the Lagrangrian procedure outlined above. This is done by calculating the dissipation function and kinetic energy corresponding to the parabolic velocity profits associated with Poiseuille flow. The viscodynamic operator thus derived is

$$
\bar{Y}(p)=\left(3 \eta / a^{2}\right)+(6 / 5) \rho_{f} p,
$$

which may be written

$$
\bar{Y}(p)=\left(3 \eta / a^{2}\right)\left(1+\frac{2}{5} z^{2}\right) .
$$

From the numerical value of $F_{1}$ it is found that in the range $0^{-} z-\sqrt{3}$ it may be represented with excellent accuracy by the approximation

$$
F_{1}=1+(1 / 15) z^{2} .
$$

For $z=\sqrt{3}$ the error is of the order of $5 \%$. With this approximation the viscodynamic operator (3.15) coincides with the value (3.17) derived by the Lagrangian method. Even in the range $\sqrt{3}-z-2 \sqrt{3}$ the error does not become significant.

\footnotetext{
11 The real and imaginary values of $F_{1}$ are plotted in Fig. 2 of reference 6 . Due to a misprint, the abscissa in that figure represents $\kappa_{1}(8 / 3)^{\frac{1}{2}}$ instead of $\kappa_{1}\left[\kappa_{1}=a(\omega / \nu)^{1}\right]$.
}

The approximate operator (3.17) may also be derived by expanding the exact expression (3.14) in powers of $z^{2}$ and limiting this expansion to the first term is $z^{2}$.

The same calculation has been repeated for the three-dimensional flow in a tube of circular cross section using the solution derived in the earlier paper. ${ }^{6}$ Again we are led to the same conclusion that the viscodynamic operator derived by the Lagrangian method and a Poiseuille microvelocity configuration is valid up to frequencies where inertia and viscous forces are of the same order. This frequency corresponds to a value $|z| \approx \sqrt{3}$ and may be used to define a transition frequency separating a "low"- and "high"-frequency range. With a pore size $2 a=10^{-2} \mathrm{~cm}$ and water at $15^{\circ} \mathrm{C}$ $\left(\nu=0.013 \mathrm{~cm}^{2} / \mathrm{sec}\right)$, the transition frequency is of the order of $300 \mathrm{cps}$.

\section{VISCODYNAMIC OPERATOR IN THE HIGHER FREQUENCY RANGE}

\section{A. Viscosity Correction Factor}

An approximate evaluation of the viscodynamic operator in the high-frequency range was made $^{6}$ by the use of a complex correction factor. This amounts to replacing the viscosity $\eta$ by a corrected complex viscosity

$$
\bar{\eta}=\eta F\left(\beta \omega / \omega_{c}\right),
$$

where $\omega_{c}$ is a characteristic frequency which may be put equal to

$$
\omega_{c}=\eta / k \rho_{f}=\nu / k,
$$

and $\beta$ is a coefficient which depends on the geometry of the pores. The correction factor $F\left(\beta \omega / \omega_{c}\right)$ is a complex function of the frequency, which is plotted in Fig. 4 of a previous paper ${ }^{6}$ as a function of $\kappa=\left(\beta \omega / \omega_{c}\right)^{\frac{1}{2}}$. We have shown that a change in the cross section of the pores may be taken into account by changing the frequency scale in the function $F$, i.e., by varying the coefficient $\beta$. It was found that for a capillary channel of cross sections resembling a narrow slit we must put $\beta=8$, while for a tube of circular cross section $\beta=16 / 3$. It can be seen that the dependence on the geometry in this case is not very sensitive. It may be assumed that in many applications other types of geometries may be incorporated approximately in the factor $\beta$.

With this correction factor the viscodynamic operator is

$$
\bar{Y}(p)=\bar{\eta} / k+m p .
$$

The inertia parameter $m$ may be evaluated from the kinetic energy. A suitable microvelocity field must be chosen. At low frequency, accurate results are obtained by using the Poiseuille flow field. As the frequency increases the microvelocity field changes gradually to a potential flow where the effect of the viscosity is confined to a thin boundary layer in the vicinity of the solid. A refined value of $m$ is obtained by taking into 
account this gradual change in the evaluation of the kinetic energy. In the case of the exact operator (3.14) it can be seen that the value of $m$ over the whole frequency range varies from $(6 / 5) \rho_{f}$ to $\rho_{f}$.

The correction factor $F$ is unity at zero frequency. As the frequency increases it becomes asymptotic to a complex value with a magnitude proportional to $(\omega)^{\frac{1}{2}}$ and a phase angle of $45 \mathrm{deg}$. This behavior is due to the fact that beyond the transition frequency the fluid friction tends to be concentrated near the solid in a boundary layer whose thickness decreases like $\omega^{-\frac{1}{2}}$. As a consequence the magnitude of the friction is higher than that which would occur if the microvelocity field retained its Poiseuille flow configuration, and is represented by an effective viscosity which instead of being constant is proportional to $(\omega)^{\frac{1}{2}}$.

\section{B. Scaled-Model Test}

Another possibility is to use model tests. From the similarity law of unsteady small motion of a viscous incompressible fluid, it can be seen that a general form of the viscodynamic operator is

$$
\bar{Y}(p)=\rho_{f} \omega_{c} \bar{f}\left(p / \omega_{c}\right) .
$$

We may choose the characteristic frequency $\omega_{c}$ to be the same as defined above by Eq. (4.2). In that case $\bar{f}=1$ at zero frequency. The function $\bar{f}\left(p / \omega_{c}\right)$ depends only on the geometry of the pores. We note that $k$ is proportional to the square of the dimensions. Hence $\omega_{c}$, which determines the frequency scale, is directly proportional to the fluid viscosity and inversely proportional to the square of the dimension.

In the particular case of plane flow between parallel boundaries the viscodynamic operator in the lowfrequency range was found to be expressed by Eq. (3.16). It may be written in the form

$$
\bar{Y}(p)=\rho_{f} \omega_{c}\left(1+\frac{6}{5} \frac{p}{\omega_{c}}\right)
$$

with a characteristic frequency

$$
\omega_{c}=3 \eta / \rho_{f} a^{2}=3 v / a^{2} .
$$

The operator (4.5) is a particular case of the general expression (4.4). Since $p=i \omega$, it shows that inertia and viscous forces are of the same order when $\omega \cong \omega_{c}$.

As an example of scaling, consider a porous medium constituted by a stacking of spheres of diameter equal

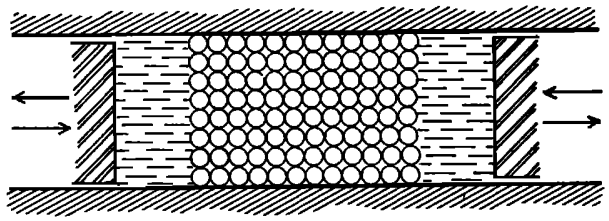

FIG. 2. Schematic scaled model for empirical evaluation of the viscodynamic operator. to $1 \mathrm{~cm}$, surrounded by oil 100 times more viscous than water. By means of a piston arrangement or some suitable driving device illustrated schematically in Fig. 2, let us apply an alternating motion to the fluid at a frequency $3 \mathrm{cps}$. This corresponds to a frequency of $300 \mathrm{cps}$ for spheres whose dimension is reduced to $1 / 10 \mathrm{~mm}$ and surrounded by water. Measurement of the amplitude and phase of the pressure in the model at various frequencies yields the function $\bar{f}$.

Such model tests may of course be used to determine the viscodynamic operator in both low- and highfrequency ranges. However, the frequency chosen for the model should be low enough so that the solid plays the role or a rigid body while the fluid may be considered as incompressible.

In applying the similarity law we should keep in mind that expression (4.4) is valid only for small amplitudes of the particle velocities. As the amplitude increases, nonlinear effects may enter into play which modify the configuration of the velocity field. Such an amplitude dependence is determined by the Reynolds number expressed in terms of the particle velocity. In the acoustic propagation theory it is generally assumed that amplitudes are small enough so that the influence of the Reynolds number is negligible.

The model test described above provides a method to determine the velocity amplitudes at which the linearity breaks down. It should be noted that for a given energy density of the acoustic wave, the fluid velocity, and therefore the Reynolds number, does not vary appreciably with frequency.

\section{Lagrangian Method}

The use of Lagrangian equations is not restricted to the low-frequency range. However, at increasing frequencies the microvelocity field is not a fixed configuration proportional to the volume flow.

Consider, for instance, a vector $\mathbf{w}$ of magnitude $w_{x}$ oriented in the $x$ direction. The Cartesian components $v_{2}$ of the microvelocity field may be written

$$
v_{i}=\alpha_{i l} q_{l},
$$

where $q_{l}$ are any numbers of generalized coordinates, each of which is associated with a fixed microvelocity configuration $\alpha_{i l}$. The volume displacement per unit area is obtained by integration and is written

$$
w_{x}=\beta_{l} q_{l} .
$$

The dissipation function and kinetic energy are the quadratic forms

$$
D=\frac{1}{2} D_{l k} \dot{q}_{l} \dot{q}_{k}, \quad \tau=\frac{1}{2} T_{l k} \dot{\dot{q}}_{l} \dot{q}_{k},
$$

and the Lagrangian equations in the relative motions are written

$$
\beta_{l}\left(-\partial p_{f} / \partial x-\rho_{f} \ddot{u}_{x}\right)=D_{l k} \dot{q}_{k}+T_{l k} \ddot{q}_{k} .
$$


Solving these equations operationally we write

$$
\dot{q}_{k}=\left(-\partial p_{f} / \partial x-\rho_{f} \ddot{u}_{x}\right) Z_{k l} \beta_{l} .
$$

Substitution of these solutions in Eq. (4.8) yields the following relation for the viscodynamics operator:

$$
1 / \bar{Y}=\beta_{k} \beta_{l} \bar{Z}_{k l} \text {. }
$$

This method is very flexible and reflects the power of the variational procedure. For example, normal coordinates may be chosen to represent the microvelocity field. In this case the matrices of $D$ and $T$ are diagonalized and Eq. (4.10) may be solved immediately. This yields the value of $1 / \bar{Y}$ directly as an expansion in partial fractions. The use of normal coordinates in the case of plane flow illustrated in Fig. 1 is equivalent to an expansion of the velocity profile in a Fourier series. The expression for $\beta_{k} \beta_{l} \bar{Z}_{k l}$ is diagonalized and turns out to be the partial fraction expansion of the corresponding transcendental function $1 / \bar{Y}$ where $\bar{Y}$ is given by Eq. (3.14). This may be verified as follows:

We write Eq. (3.14) in the form,

$$
\frac{1}{\bar{Y}}=\frac{a^{2}}{3 \eta} \frac{3}{z^{2}}\left(1-\frac{1}{z} \tanh z\right) \text {. }
$$

Using Laurent series expansions we derive the identities

$$
\begin{aligned}
\frac{1}{z} \tanh z & =2 \sum_{k=0}^{\infty} \frac{1}{z^{2}+(2 k+1)^{2} \frac{1}{4} \pi^{2}}, \\
1 & =2 \sum_{k=0}^{\infty} \frac{1}{(2 k+1)^{2} \frac{1}{4} \pi^{2}} .
\end{aligned}
$$

$$
\frac{1}{\bar{Y}}=\frac{a^{2}}{3 \eta} \frac{24}{\pi^{2}} \sum_{k=0}^{\infty} \frac{1}{(2 k+1)^{2}\left[z^{2}+(2 k+1)^{2} \frac{1}{4} \pi^{2}\right]} .
$$

This corresponds to the general form (4.12) of the viscodynamic operator where normal coordinates are used. For values of $z$ that are not too large, the series is rapidly convergent. If we limit ourselves to the first term $(k=0)$ we find

$$
\bar{Y}=\left(3 \eta / a^{2}\right)\left(1.01+0.412 z^{2}\right),
$$

a value very close to the one derived above (3.17). This example justifies the conclusion that the use of normal viscodynamic coordinates will, in general, lead to rapidly convergent series for frequencies up to two or three times the transition range. The physical reason for this resides in the property that at a given frequency predominant excitation occurs only for the viscodynamic modes whose characteristic relaxation times are larger than the period of the oscillation.

\section{ANISOTROPIC MEDIA}

Propagation equations for anisotropic media are immediately derived by direct application of the foregoing methods.
The stress-strain relations for an elastic porous matrix in the most general case of anisotropy are written

$$
\begin{aligned}
\tau_{i j} & =A_{i j}{ }^{\mu \nu} e_{\mu \nu}+M_{i j} \zeta, \\
p_{f} & =M_{i j} e_{i j}+M \zeta .
\end{aligned}
$$

They contain 28 elastic coefficients. Such equations were derived and discussed in previous work. ${ }^{10,12}$

The dynamical equation (2.2) remains the same and Eq. (2.3) is replaced by

$$
-\partial p_{f} / \partial x_{i}-\rho_{f} \ddot{u}_{i}=\bar{Y}_{i j}(p) \dot{w}_{j} .
$$

The viscodynamic operator $\bar{Y}_{i j}$ is now a symmetric tensor of the second rank. Its components are complex quantities, functions of the frequency. Evaluation of this operator follows the same procedure as outlined in Secs. III and IV. Formulation of the problem by the Lagrangian equations in paragraph (c) of Sec. IV leads to the conclusion that the viscodynamic tensor is symmetric.

In the low-frequency range we may use the microvelocity field given by the Poiseuille flow. The dissipation function and kinetic energy then take the form

$$
\begin{aligned}
& D=\frac{1}{2} \eta r_{i j} \dot{w}_{i} \dot{w}_{j}, \\
& \tau=\frac{1}{2} m_{i j} \dot{w}_{i} \dot{w}_{j},
\end{aligned}
$$

and the viscodynamic operator is written

$$
\bar{Y}_{i j}(p)=\eta r_{i j}+m_{i j} p
$$

In the case where the fluid dissipation is not determined entirely by the viscosity but depends on more complex interfacial phenomena, a more general coefficient $R_{i j}$ must be written instead of $\eta r_{i j}$.

In the case of orthotropic symmetry we choose the coordinate axes to lie in the planes of symmetry. They coincide with the principal directions of the viscodynamic tensor. We may write

$$
\bar{Y}_{i j}=\left[\begin{array}{ccc}
\bar{Y}_{x x} & 0 & 0 \\
0 & \bar{Y}_{y y} & 0 \\
0 & 0 & \bar{Y}_{z z}
\end{array}\right] .
$$

The diagonal components are of the form

$$
\bar{Y}_{x x}=\rho_{f} \omega_{x} \bar{f}_{x}\left(p / \omega_{x}\right) .
$$

This introduces three characteristic frequencies $\omega_{x}, \omega_{y}$, $\omega_{z}$ corresponding to the principal directions. They are defined by expressions of the type (4.2) in terms of three principal permeability coefficients. When using the function $F$ of Eq. (4.1), an approximation is derived by using the same function with a different frequency scale in each principal direction. These frequency scales are determined by three values of $\omega_{c}$ and $\beta$ attached to each principal direction.

We note that for cubic symmetry the tensor $\bar{Y}_{i j}$ is isotropic.

12 M. A. Biot, "Theory of Elasticity and Consolidation for a Porous Anisotropic Solid," J. Appl. Phys. 26, 182-185 (1955). 


\section{VISCOELASTICITY AND SOLID DISSIPATION}

When dealing with actual materials, the model of a purely elastic matrix saturated with a viscous fluid will only be applicable in exceptional cases. The range of relaxation phenomena associated with dissipative properties in porous solids extends to the whole field of physics and chemistry. The porous structure introduces a large area of contact of fluid and solid and in many cases interfacial surface phenomena will play a dominant role. Therefore, it is not sufficient to take into account the separate physical properties of fluid and solid, but the porous medium must be considered as a whole. For example, consider the case where the fluid is an electrolyte in which the components of the solid matrix may enter into solution. When the system is at rest its state of equilibrium is the result of very complex interactions which involve chemical affinity, electrical potentials, Van der Waals forces, etc. When this equilibrium is disturbed, the system tends to a new state. However, the change is not instantaneous and time lags appear which reflect the existence of a relaxation spectrum.

Obviously also the state properties of the system will depend on the fluid in the pores. Because of the large areas involved, interfacial energies will contribute substantially to the over-all elastic rigidity. This may be expressed in another way by saying that in this connection the surface tension will play a significant role as already pointed out by this writer many years ago. ${ }^{7}$ If the pore fluid is removed by evaporation, not only will the surface phenomena be drastically affected but the components in the solution will precipitate, thereby modifying the gap size in the areas of minute cracks and intergranular regions. In addition, the surface tension effects will be associated with their own relaxation times.

These microscopic gaps must be distinguished from the pores proper which are of much larger size and where the main fluid motion occurs. More generally, the physical chemistry of microscopic domains of fluid in cracks and intergranular gaps should be an important factor in determining the over-all rigidity and dissipation in the viscoelastic porous matrix. Fluids under these conditions and especially at the higher frequencies will exhibit rigidity and relaxation effects due to complex surface and intermolecular forces which are quite different from classical Newtonian viscosity.

Under these conditions large variations in applied effective pressure which affects the size of the gaps should induce appreciable changes in the acoustic properties. Moreover, the parameters which govern this dependence will involve the physical chemistry of the multiphase system.

Regions of intergranular contact also contribute to the rigidity and dissipation. Such regions exhibit all the gradations between an amorphous and crystalline structure with a complex array of dislocations. At small acoustic amplitudes relaxation phenonena in these regions should predominate and the assumption of linearity will be justified.

Thermoelastic dissipation is another example of interactions between fluid and solid phases. Stress waves set up temperature differences in the fluid and the solid, and this initiates interfacial heat flow associated with a relaxation time spectrum.

In addition to these fluid-solid interactions, the solid and the fluid themselves exhibit relaxation properties. For the solid this is the result of what is usually referred to as solid dissipation.

The fluid itself may cause dissipation because of bulk relaxation, the appearance of a time lag between the changes of pressure and volume. This is observed in water at certain frequencies when specific salts are in solution.

In spite of the enormous diversity of all these phenomena it is possible to formulate a general theory which take these effects into account. Such a phenomenological approach was developed by the writer based on an extension of the principle of nonequilibrium thermodynamics. It is in such broader context that we have used the term viscoelasticity.

In this context the porous medium is considered to be defined by a certain number of thermodynamic variables which include the "observed" mechanical coordinates and the "hidden" coordinates. Here an example of hidden coordinates would be the physicalchemical parameters defining the thermodynamic state of microscopic domains including large surface effects.

Knowledge of the generalized free energy of this system in terms of all these variables yields the elastic coefficients. If in addition the dissipation function can be determined in terms of the time derivatives of these variables, the time history and relaxation effects may be evaluated.

Elimination of the hidden coordinates from the equations provides operational relations between the observed strain and stress components. Apparent frequency-dependent rigidity coefficients will appear as a result of the progressive "freezing" of the hidden degrees of freedom with increasing frequency.

The thermodynamic relaxation theory and its extension to porous media were developed in two papers. ${ }^{12,13}$ This work brings forth a general correspondence principle by which known results for elastic media may be immediately extended to the case of viscoelasticity by substituting operators for the elastic coefficients. It is applicable to porous and nonporous media, anisotropy, dynamics, and variational methods.

By this correspondence principle the stress-strain relations for a porous medium with relaxation and viscoelastic properties are formally identical. In the

\footnotetext{
${ }^{13}$ M. A. Biot, "Theory of Stress-Strain Relations in Anisotropic Viscoelasticity and Relaxation Phenomena," J. Appl. Phys. 25, 1385-1391 (1954).
} 
case of isotropy substituting operators for the elastic coefficients in Eq. (2.1), we write

$$
\begin{aligned}
\tau_{i j} & =2 \bar{\mu} e_{i j}+\delta_{i j}(\bar{\lambda} e-\bar{C} \zeta), \\
p_{f} & =-\bar{C} e+\bar{M} \zeta .
\end{aligned}
$$

The coefficient $\alpha M$ has been replaced by the operator $\bar{C}$. The thermodynamic theory leads to a general expression for these operators. For example, the shear operator $\bar{\mu}$ may be written

$$
\bar{\mu}=\int_{0}^{\infty} \frac{p}{p+r} \mu(r) d r+\mu+\mu^{\prime} p .
$$

The symbol $p$ denotes the time derivative (2.4) or $i \omega$ (2.5). The propagation equations (2.6) for a uniform isotropic medium become

$$
\begin{aligned}
\bar{\mu} \nabla^{2} \mathbf{u}+\operatorname{grad}\left[\left(\bar{\mu}+\bar{\lambda}_{c}\right) e-\bar{C} \zeta\right] & =\frac{\partial^{2}}{\partial t^{2}}\left(\rho \mathbf{u}+\rho_{f} \mathbf{w}\right), \\
\operatorname{grad}(\bar{C} e-\bar{M} \zeta) & =\frac{\partial^{2}}{\partial t^{2}}\left(\rho_{f} \mathbf{u}\right)+\bar{Y} \frac{\partial \mathbf{w}}{\partial t} .
\end{aligned}
$$

Similarly, equations for the propagation in anisotropic media are immediately derived by substituting operators in Eq. (5.1).

Attention is called to the possibility of including nonlinear dissipation effects of intergranular contact areas at the higher amplitudes by introducing empirically a gradual amplitude dependence of the modulus and phase of the operators with increasing amplitudes.

\section{ILLUSTRATIVE VISCOELASTIC MODELS}

As an illustration we shall briefly discuss some examples of such operators. The examples are presented in the context of mechanics. However, they may be considered as analogue models for some of the more complex physical-chemical phenomena discussed in the preceding section.

Solid dissipations in shear may be represented by putting

$$
\bar{\mu}=a p^{s}+\mu .
$$

Physically this operator represents two elements in parallel. One is purely elastic of modulus $\mu$. The other represents creep of such a nature that the deformation under constant stress is proportional to $t^{8}$ ( $t=$ time). When $0<s<1$ we recognize a type of creep frequently observed in solids, and similar to one derived from dislocation theory.

Let us assume $s$ to be small and consider the case of acoustic propagation at angular frequency $\omega$. We write approximately

$$
\bar{\mu}=a \omega^{s}\left(1+\frac{1}{2} i \pi s\right)+\mu .
$$

The imaginary part varies slowly with frequency. Since it is proportional to the logarithmic decrement of the material, we see that the operator (7.2) may approximate a type of solid dissipation frequently observed..$^{14}$ For acoustic propagation a constant decrement yields an absorption coefficient proportional to the frequency.

As shown elsewhere ${ }^{9}$ the operator (7.1) may be written in the general form (6.2) by introducing a relaxation spectrum

$$
\mu(r)=a(\sin s \pi / \pi) r^{8-1} .
$$

Another operator of similar property proposed earlier by this writer ${ }^{9}$ is obtained by putting

$$
\mu(r)=\left\{\begin{array}{cc}
\mu_{1} / r & r>\epsilon, \\
0 & r<\epsilon .
\end{array}\right.
$$

With $\mu^{\prime}=0$ and $p=i \omega$, Eq. (6.2) yields

$$
\bar{\mu}=\mu_{1}\left(\frac{\pi}{2}-\tan ^{-1} \frac{\epsilon}{\omega}\right) i+\mu_{1} \log \left(1+\frac{\omega}{\epsilon}\right)^{\frac{1}{2}}+\mu .
$$

For a value of $\epsilon$ suitably small the imaginary term is approximately independent of the frequency.

Interfacial dissipation may be illustrated by some simple examples. As already pointed out, physical chemistry will generally have to be brought into the picture. Purely mechanical effects come readily to our attention if we consider the areas of contact between grains. Such regions are usually of disordered molecular structure thereby acquiring properties analogous to a fluid of high viscosity. Such properties are represented by operators with viscous elements.

Other intergranular effects bring into play the viscosity of the fluid in the pores. A model for such a mechanism is illustrated in Fig. 3(a) which represents two elastic grains in contact in the presence of a fluid. If an oscillating stress is applied normally to the area of contact, the fluid will be squeezed in and out of the narrow regions $A$ and $B$. Because of the fluid viscosity this produces a dissipation. A spring-dashpot model corresponding to this mechanism is shown in Fig. 3(b). The operator representing this model is given by Eq. (7.17). A similar dissipation mechanism occurs

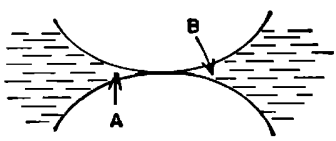

(a)

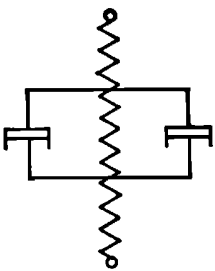

(b)
FIG. 3. Schematic representation of the region of contact between grains and the corresponding spring-dashpot model.

${ }^{14}$ L. Peselnick and W. F. Outerbridge, "Internal Friction in Shear and Shear Modulus of Solenhofen Limestone over a Frequency Range of $10^{7}$ Cycles per Second," J. Geophys. Research 66, 581-588 (1961). 

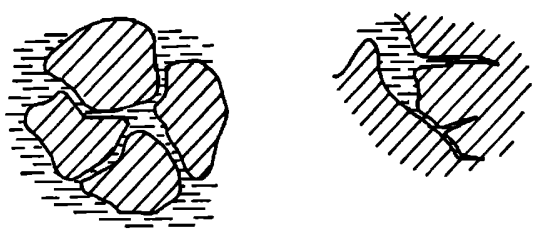

FIG. 4. Illustration of actual grain and fluid-gap configurations.

when the pores include narrow intergranular gaps or wall cracks of a type illustrated in Fig. 4.

It is of interest to evaluate the type of operators associated with such a mechanism when the fluid compressibility is taken into account. This can be done by considering a simplified model represented by a small gap of fluid between two rigid blocs as shown in Fig. 5. The model is two-dimensional. The $x$ coordinate is oriented along the length of the gap. The width of the gap is $h$. The relation between the fluid pressure and the average displacement $w$ of the fluid over the width of the gap is obtained from the viscodynamic operator (3.16) after putting $p=0$. We derive,

$$
-\frac{\partial p_{f}}{\partial x}=\frac{12 \eta}{h^{2}} \frac{\partial w}{\partial t},
$$

where $\eta$ denotes the fluid viscosity. On the other hand, the fluid elasticity yields the equation

$$
-c p_{f}=\partial w / \partial x
$$

where $c$ is the fluid compressibility. Combining the two equations (7.6) and (7.7) yields

$$
\frac{\partial^{2} p_{f}}{\partial x^{2}}=\frac{12 \eta c}{h^{2}} \frac{\partial p_{f}}{\partial t} .
$$

An initial pressure distributed as a half sine wave over the length $D$ of the gap will decay exponentially with time. This is represented by the following solution of Eq. (7.8):

$$
p_{f}=p_{1} \sin (\pi x / D) e^{-r t}
$$

with a relaxation time

$$
1 / r=12 \eta c D^{2} / \pi^{2} h^{2} \text {. }
$$

For a gap of dimensions

$$
\begin{aligned}
D & =10^{-1} \mathrm{~cm}=1 \mathrm{~mm}, \\
h & =10^{-4} \mathrm{~cm}=1 \mu,
\end{aligned}
$$

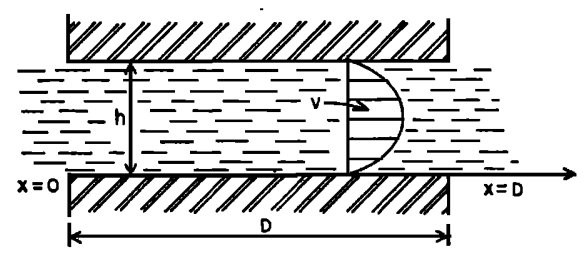

FIG. 5. Simplified model for a compressible fluid gap between solid boundaries. filled with water at $20^{\circ} \mathrm{C}$, the relaxation time is

$$
1 / r=5.7 \times 10^{-7} \mathrm{sec} \text {. }
$$

This corresponds to a frequency of about $2 \mathrm{Mc}$. At this frequency non-Newtonian viscoelastic behavior of the fluid must be considered. In addition the wavelength begins to approach the pore size and wave scatter must be taken into account. Dissipation due to scatter is not within the scope of the present theory. For a gap $h=10^{-5} \mathrm{~cm}$ the relaxation frequency is brought down to the $20 \mathrm{kc}$ range. However, as already pointed out, we must bear in mind that the behavior of water in gaps of this size or smaller may depart from that of a Newtonian fluid, due to strong surface effects.

Actually, of course, there is a spectral distribution of such relaxation times corresponding to all the possible sine wave distributions of pressure across the gap. The relaxation times of these spectral components are proportional to $1,1 / 9,1 / 25$, etc. The gap is therefore equivalent to a number of Maxwell elements in parallel as shown in Fig. 6 . The force $P$ acting normally

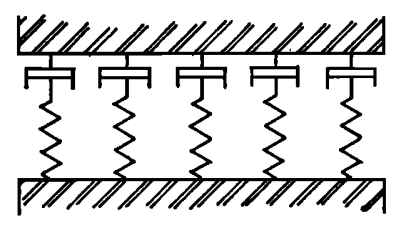

Fig. 6. Maxwell elements in parallel equivalent to the fluid gap of Fig. 5.

to the gap and the change of width of $\Delta h$ are related operationally by an expression of the type

with

$$
P=\bar{Z} \Delta h
$$

$$
\bar{Z}=\frac{a_{1} p}{p+r_{1}}+\frac{a_{2} p}{p+r_{2}}+\cdots .
$$

As an example let us show how such an element can be introduced into the evaluation of the operators of the general equations (6.1). A solid elastic column with a fluid-saturated crack may be represented schematically by a block diagram of elastic and viscoelastic elements as shown in Fig. 7. The jacketed compressibility operator corresponding to this model is

$$
\bar{\kappa}=\kappa_{0}+\frac{1}{1 / \kappa_{1}+\bar{Z}} .
$$

At a frequency low enough with respect to $r_{1}$ the expression for $\bar{Z}$ may be reduced to the approximation

$$
\bar{Z}=\left(a_{1} / r_{1}\right) p,
$$

which represents a purely viscous element. The compressibility operator then becomes

$$
\bar{\kappa}=\kappa_{0}+\kappa_{1} p /\left(r_{1}+a_{1} \kappa_{1} p\right) \text {. }
$$

This corresponds to the spring-dashpot model of Fig. 3(b). 

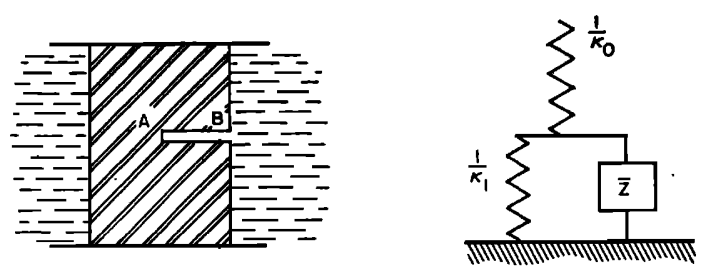

FIG. 7. Fluid-saturated crack $A B$ in a solid, and its equivalent block diagram.

Using a correspondence principle we have shown ${ }^{9}$ how the operators of the stress-strain relation (6.1) could be evaluated. For example, the operators $\bar{C}$ and $\bar{M}$ are

$$
\begin{gathered}
\bar{C}=\frac{1-\delta / \bar{\kappa}}{\gamma+\delta-\delta^{2} / \bar{\kappa}}, \\
\bar{M}=\frac{1}{\gamma+\delta-\delta^{2} / \bar{\kappa}} .
\end{gathered}
$$

The unjacketed compressibility $\delta$ and the coefficient of fluid content $\gamma$ were introduced and discussed earlier. ${ }^{15}$ They are defined by considering a unit volume of bulk material in an unjacketed test. A unit increase of fluid pressure $\delta$ represents the change of volume of the bulk solid while $\gamma$ is the volume of fluid entering the solid through the pores.

Operators of the type (7.18), corresponding to a model of fluid-saturated cracks or integranular gaps, were also discussed in a previous paper ${ }^{10}$ in the more general context of the thermodynamics of irreversible processes and as a particular example of the more general theory.

Attention has also been called ${ }^{9,15}$ to the possibility of expressing $\gamma$ in terms of the porosity $f$ under certain assumptions regarding the structure of the porous solid. This expression is

$$
\gamma=f(c-\delta),
$$

where $c$ is the fluid compressibility.

The assumption that $\gamma$ and $\delta$ are real quantities is, of course, not necessary. Bulk relaxation can be introduced if we replace $\gamma$ and $\delta$ by the corresponding operators. This may be illustrated by the case where bulk viscosity or relaxation of the fluid is taken into account. Various relaxation processes have been suggested. In one of the models discussed by Hirai and Eyring $^{16}$ the bulk viscosity in water results from a shifting equilibrium between the number of holes and the number of phonons associated with the liquid lattice. This leads to a relaxation time in the pressurevolume relationship and corresponds to the mechanical model of Fig. 7 where $\bar{Z}$ is a viscous element. The

${ }^{16}$ M. A. Biot and D. G. Willis, "The Elastic Coefficients of the Theory of Consolidation," J. Appl. Mech. 24, 594-601 (1957).

${ }^{16}$ N. Hirai and H. Eyring, "Bulk Viscosity of Liquids," J. Appl. Phys. 29, 810 (1958). fluid compressibility is then represented by an operator of the type (7.17), i.e.,

$$
\bar{c}=c_{0}+c_{1} r /(r+p) .
$$

In such a fluid there will be a transition range where the acoustic velocity changes while the dissipation goes through a peak. The same phenomenon will also occur for certain salt solutions in water. In this case the shifting equilibrium corresponds to molecular changes in the solution. Again this will be represented by a compressibility operator of the type (7.20). Introduction of a compressibility operator $\bar{c}$ for the fluid replaces $\gamma$ by an operator $\bar{\gamma}$ in the previous formulas.

We have also discussed ${ }^{9}$ a type of dissipation due to air bubbles. This however may involve resonance effects since the air in the bubble and the apparent mass of the surrounding liquid combine to form an oscillator. The fluid is then represented by the mechanical system of Fig. 8. It differs from those considered above by the addition of a mass element $m$. The compressibility operator by the fluid in this case becomes

$$
\bar{c}=c_{2}+1 /\left(m p^{2}+\beta p+k\right) .
$$

This operator does not belong to the general type (6.2) considered previously because it takes into account an inertia effect in the hidden coordinates. This appears in the term $m p^{2}$. The damping term $\beta p$ corresponds to the viscous dissipation arising from the concentrated radial velocity of the fluid in the vicinity of the bubble. A thermoelastic dissipation is also associated with the presence of gas bubbles. It may be introduced in the present formulation by the substitution of an appropriate operator $\bar{k}$ in the place of the elastic coefficient $k$.

Another viscoelastic model is illustrated by the mechanical properties associated with the physical and chemical equilibria at the boundary between the solid and the fluid in the pores. Because of the large area of the fluid-solid interface, such effects usually play a significant part in determining the over-all properties of the bulk material. The so-called surface tension effects belong to this class of phenomena. The shift in physical and chemical equilibria at the interface will generally involve relaxation times which may be evaluated by the method of statistical mechanics. The operators representing such effects will be of the same general type as those already considered. For example,

Fig. 8. Spring, mass, and dashpot model representing a fluid containing bubbles.

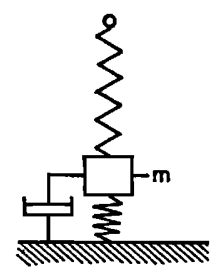


the shear operator $\bar{\mu}$ in such a case will be given by

$$
\bar{\mu}=\mu_{0}+[p /(r+p)] \mu_{1} .
$$

Such an operator exhibits the characteristic features of a transition frequency with a dissipation peak. The presence of a spectrum of relaxation effects may be taken into account by adding a number of terms of the same type in Eq. (7.22).

\section{THERMOELASTIC AND ELECTROKINETIC DISSIPATION}

When considering thermoelastic relaxations in heterogeneous media, two types of phenomena must be distinguished. One type of thermoelastic dissipation is due to the heterogeneities. In a porous medium this is represented by the microscopic heat flow taking place between small domains of thermoelastic temperature differences within the solid matrix and between the pore-fluid and the solid. Attention is called to the analogous case of nonuniform porosity, where unequal fluid pressures are generated and play the same role as unequal temperatures in thermoelasticity. Such effects are included in the general type of operational expressions such as (6.2). The particular operators for a specific geometry may be derived by using the general thermodynamic theory. ${ }^{10}$ The other type of thermoelastic effect is due to the over-all temperature gradients generated by the acoustic wave and will generate a thermoelastic dissipation analogous to the case of a continuum.

A word should also be said about electrokinetic effects. The relative motion of the fluid in the pores generates an electric field and electric currents. These currents contribute to the acoustic dissipation. The electrokinetics of porous membranes is well known to physical chemists. In the light of the present theory it is possible to distinguish two types of electrokinetic effects which resemble the distinction between the two types of thermoelastic dissipation mentioned above. In one case the relative motion of the fluid is purely local and is due to the squeezing of the fluid in and out of cracks and narrow gaps as in the various examples which we have discussed. This generates local dipoles. For isotropic porosity these dipoles and the local currents which they generate will exhibit a certain degree of randomness. In such a case the present theory is applicable by considering these local dipoles to represent "hidden" degrees of freedom. This type of electrokinetic effect is included in the present thermodynamic treatment and may be incorporated in the viscoelastic operators. The other and more important case considers the electric potential associated with the over-all relative fluid velocity. It is possible to calculate this effect by evaluating the relative fluid velocity from the present theory. The corresponding electric potentials and currents may then be derived. In this case the currents are not random but they are distributed sinusoidally with the same wavelength as the acoustic wave. A discussion of the electrokinetic effect in the context of acoustic propagation was already given by Frenkel ${ }^{1}$ for the low-frequency range. Actually there is a coupling between the electric field and the pressure gradient. A more rigorous treatment including such coupling involves the Onsager relations and the same thermodynamic principles which have been discussed above in the context of viscoelasticity. Application of the thermodynamic theory to this case will result in equations for the coupled fields of electrical and mechanical variables. An interesting aspect of this phenomenon is the relative importance assumed by the wave of the second kind as compared to the wave of the first kind. We have shown that the wave of the second kind is associated with a larger relative velocity of the fluid in the pores. ${ }^{6}$ This should have an important bearing on the electrokinetic effect. 